\title{
A local limit theorem for a sequence of interval transformations
}

\author{
P. CALDERONI, M. CAMPANINO AND D. CAPOCACCIA \\ ZiF, Universität Bielefeld, 4800 Bielefeld, West Germany; Instituto Matematico \\ 'G. Castelnuovo', Università di Roma, 00185 Roma, Italy
}

(Received 2 November 1984 and revised 28 November 1984)

Abstract. Let $\lambda>1$ be a real eigenvalue of an automorphism of the two dimensional torus. We prove that for a dense, open subset of intervals $[a, b] \subset[0,1]$, the sequence $\nu_{N}(x)=\sum_{k=0}^{N-1} \chi_{[a, b]}\left(\left\{\lambda^{k} x\right\}\right)$, where $\{x\}$ denotes the fractional part of $x$ and $\chi_{[a, b]}$ the characteristic function of $[a, b]$, satisfies the local limit theorem with respect to Lebesgue measure on $[0,1]$.

\section{0 . Introduction}

Let $\lambda$ be a real number, $\lambda>1$. It is known (see e.g. [3, p. 164]) that for almost every $x \in[0,1]$, with respect to the Lebesgue measure, the sequence $\left\{\lambda^{k} x\right\}, k \geq 0$, where $\{x\}$ denotes the fractional part of $x$, is uniformly distributed in $[0,1]$ : that is, if $\chi_{[a, b]}$ denotes the characteristic function of a proper subinterval $[a, b] \subset[0,1], l$ the Lebesgue measure in $[0,1]$ and $\nu_{N}$ the random variable $\nu_{N}(x)=\sum_{k=0}^{N-1} \chi_{[a, b]}\left(\left\{\lambda^{k} x\right\}\right)$,

$$
\lim _{N \rightarrow \infty} \nu_{N}(x) / N=b-a, \quad \text { for } l \text { a.e. } x .
$$

We say that the local limit theorem holds if the probability distribution of the normalized fluctuations of $\nu_{N}$ tends, as $N \rightarrow \infty$, to the normal distribution in the following strong sense:

(i) $\lim _{N \rightarrow \infty} D_{i}^{N} / N=\sigma^{2}>0$;

(ii) $\lim _{N \rightarrow \infty} \sup _{0 \leq k \leq N}\left(\sqrt{D_{l}^{N}} P_{l}^{N}(k)-\exp \left(-\left(k-E_{l}^{N}\right)^{2} / 2 D_{l}^{N}\right) / \sqrt{2 \pi}=0\right.$;

where $E_{l}^{N}, D_{l}^{N}, P_{l}^{N}$ denote, respectively, the expectation, the variance and the probability distribution of $\nu_{N}$ with respect to $l$.

The local limit theorem has been proved by Moskvin and Postnikov [8] for $\lambda=2$. In this case $\left\{\lambda^{n} x\right\}=T^{n}(x)$, where $T$ is the map of $[0,1]$ to itself $T(x)=2 x \bmod 1$, that leaves $l$ invariant. In this case $(0.2)$ may also be obtained as a consequence of more general theorems about expanding maps of the interval [11].

We consider here the case of $\lambda$ being a real eigenvalue of an automorphism $\varphi$ of the two dimensional torus $T^{2}=\mathbb{R}^{2} / \mathbb{Z}^{2}$. In this case the sequence $\left\{\lambda^{n} x\right\}$ is not the orbit of $x$ for a map of $[0,1]$ to itself, but it can be regarded as the projection on one of the coordinate axes of a $\varphi$ orbit of a point $P(x) \in T^{2}$. This fact allows us to 
exploit the existence of a Markov partition for $\varphi$ to derive (0.2) from a local limit theorem for a functional on a Markov chain $S_{N}(\varepsilon)=\sum_{K=0}^{N-1} \xi\left(T^{k} \varepsilon\right)$, where $\varepsilon=$ $\left(\varepsilon_{i}\right)_{i \in \mathbb{Z}} \in \mathscr{E}=\{0, \ldots, V\}^{\mathbb{Z}}, T$ is the shift on $\mathscr{E}$ and $\xi$ is a function on $\mathscr{E}$, which, in an appropriate sense, depends weakly on the $\varepsilon_{i}$ 's with large $i$. We follow in our proof the classical method introduced by Gnedenko [5] for independent random variables and extended by Dobrushin and Tirozzi [4] to Gibbs random fields. The estimate of the difference between the distribution of a sum of random variables and the normal distribution is derived by an estimate of the characteristic function. The first part of our argument is the proof of a strong version of the integral limit theorem. Though this part of the proof is quite standard we have included it in the paper, since the results in the literature on related problems [6], [9] do not apply to our case.

The main difficulties arise in the second part of the proof, where we need to derive a lower bound on the variance of $S_{N}$ and to check an aperiodicity condition on its values in order to get an upper bound on the characteristic function of $S_{N}$. We remark that these properties are usually given as hypotheses in the literature about local limit theorems.

The difficulties are mainly due to the fact that not all the transitions between symbols are allowed and the variable $\xi$ depends on all the symbols $\varepsilon_{i}$. We therefore consider only a subset of intervals $[a, b] \subset[0,1]$. This subset is an open, dense set containing all the intervals $[a, b]$ such that either $a$ or $b$ is sufficiently near to the first coordinate of a homoclinic point of the automorphism $\varphi$. In a subset of probability space, which asymptotically in $N$ has full measure, the problem of the long range dependence of the variable $\xi$ can be handled. Using special compatible sequences, we prove condition $(0.2(i))$ and we check the aperiodicity condition on values of $S_{N}$. The orbit of a homoclinic point corresponding to the interval $[a, b]$ is used to construct the special compatible sequences. We conjecture that the local limit theorem is verified at least on a full measure set of intervals, as remark $(2.10)$ seems to indicate.

In $\S 1$ we recall some properties of the toral automorphisms and of the Markov partitions. These properties are used in $\$ 2$ to show how $(0.2)$ follows from a local limit theorem for a functional on a Markov chain and to derive the crucial estimates that are needed in the proof of this theorem. The theorem is proved in $\S 3 . \S 4$ is devoted to a brief review of results related to ours.

\section{Symbolic dynamics}

Let $\varphi$ be an automorphism of the two-dimensional torus $T^{2}=\mathbb{R}^{2} / \mathbb{Z}^{2}$ induced by a linear transformation $A: \mathbb{R}^{2} \rightarrow \mathbb{R}^{2}$ with integral entries, det $A=1$, eigenvalues $\lambda>1$, $1 / \lambda$ and corresponding unit eigenvectors $\underline{e}^{u}=\left(e_{1}^{u}, e_{2}^{u}\right), \underline{e}^{s}=\left(e_{1}^{s}, e_{2}^{s}\right)$. We assume $e_{1}^{u}$, $e_{2}^{u}>0$; if not we can consider the automorphism induced by $A^{\prime}=\theta A \theta^{-1}$ where

$$
\theta=\left(\begin{array}{cc}
\operatorname{sign} e_{1}^{u} & 0 \\
0 & \operatorname{sign} e_{2}^{u}
\end{array}\right)
$$

$d(\cdot, \cdot)$ will denote the Euclidean distance on $T^{2}$ and $m$ the Lebesgue measure on $T^{2}$. For $\underline{p}=\left(p_{1}, p_{2}\right) \in T^{2}, W^{u}(\underline{p})$ and $W^{s}(\underline{p})$ will denote the unstable and stable 
manifolds through $\underline{p}$ :

$$
W^{u(s)}(\underline{p})=\left\{\underline{p}+\xi \underline{e}^{u(s)}(\bmod 1) ; \xi \in \mathbb{R}\right\},
$$

and, for $\delta>0$,

$$
W_{\delta}^{u(s)}(\underline{p})=\left\{\underline{p}+\xi \underline{e}^{u(s)}(\bmod 1) ;|\xi| \leq \delta\right\} .
$$

Let $H(\underline{0})$ be the set of the homoclinic points of $\underline{0}=(0,0), H(\underline{0})=W^{u}(\underline{0}) \cap W^{s}(\underline{0})$. We note that $H(\underline{0})$ is a dense subgroup of $T^{2}$ and if $\underline{p} \in H(\underline{0})$, then $\lim _{|k| \rightarrow \infty} \varphi^{k}(\underline{p})=\underline{0}$.

In order to study the statistical properties of $\varphi$ we will use the symbolic dynamics induced by $\varphi$ via a generating Markov partition of the torus. A Markov partition $\mathscr{P}$ (see $\left[12\right.$, p. 102]) is a finite family of open parallelograms $\left(P_{0}, \ldots, P_{v}\right)$ with sides parallel to $\underline{e}^{s}$ and $\underline{e}^{u}$ such that:

(i) $\bigcup_{i=1}^{v} \bar{P}_{i}=T^{2}$;

(ii) $P_{i} \cap P_{j}=\varnothing$, for $0 \leq i, j \leq v, i \neq j$;

(iii) $\partial^{s}(\varphi(\mathscr{P})) \subset \partial^{s}(\mathscr{P})$;

(iv) $\partial^{u}(\varphi(\mathscr{P})) \supset \partial^{u}(\mathscr{P})$;

with $\partial^{s(u)}(\mathscr{P})=\bigcup_{i=1}^{v} \partial^{s(u)}\left(P_{i}\right)$, where $\partial^{s(u)} P_{i}$ denotes the contracting (expanding) boundary of $P_{i}$, i.e. the union of the sides of $P_{i}$ parallel to $\underline{e}^{s(u)}$, and analogously for $\varphi(\mathscr{P})$. The Markov partition $\mathscr{P}$ is called generating if the smallest complete (w.r.t. $m$ ) $\sigma$-algebra containing the sets $\varphi^{k}\left(P_{i}\right),-\infty<k<+\infty, 0 \leq i \leq v$, is equal to the $\sigma$-algebra of all the $m$-measurable sets of $T^{2}$. In [12, pp. 106-108] and [1] an explicit construction of a generating Markov partition $\mathscr{P}$ for an automorphism $\varphi$ is given. By considering the generating Markov partition

$$
\mathscr{P}^{(0)}=\left(Q_{0}, \ldots, Q_{u}\right)=\varphi^{-n}(\mathscr{P}) \vee \cdots \vee \mathscr{P}_{\vee} \cdots \vee \varphi^{n}(\mathscr{P}),
$$

composed of all non-empty intersections of the type $\bigcap_{k=-n}^{n} \varphi^{k}\left(P_{i_{k}}\right)$ with $n$ large enough, we easily see that for every $\delta_{0}, 0<\delta_{0}<1$, we can find a generating Markov partition $\mathscr{P}^{(0)}$ such that

$$
\begin{gathered}
\operatorname{diam} \mathscr{P}^{(0)}=\sup _{0 \leq i \leq u} \operatorname{diam} Q_{i}<\delta_{0}, \\
\inf _{0 \leq i \leq u} m\left(Q_{i}\right) \geq c \delta_{0}^{2},
\end{gathered}
$$

where $c$ is a positive constant depending only on $\varphi$. It follows from the construction of $\mathscr{P}$ that we can assume there are four parallelograms of $\mathscr{P}^{(0)}$, which we will denote by $Q_{0}, \ldots, Q_{3}$, such that $\underline{\underline{0}} \in \bar{Q}_{0} \cap \cdots \cap \bar{Q}_{3}$. We note that if $i \in\{0, \ldots, 3\}$ and $l_{1}^{(i)}$ $\left(l_{2}^{(i)}\right)$ is the length of the sides of $Q_{i}$ parallel to $\underline{e}^{u}\left(\underline{e}^{s}\right)$, then for every $k, \varphi^{k}\left(Q_{i}\right)$ is a parallelogram with a vertex in $\underline{0}$, lying 'on the same side' of $\underline{0}$ as $Q_{i}$ and with sides parallel to $\underline{e}^{u}\left(\underline{e}^{s}\right)$ of length $\lambda^{k} l_{1}^{(i)}\left(\lambda^{-k} l_{2}^{(i)}\right)$.

Given $\mathscr{P}^{(0)}$ we define a $u \times u$ matrix $B$ by:

$$
B_{i j}= \begin{cases}1 & \text { if } \varphi\left(Q_{i}\right) \cap Q_{j} \neq \varnothing, \\ 0 & \text { otherwise. }\end{cases}
$$

We denote by $\overline{\mathscr{E}}_{\text {and }} \overline{\mathscr{E}}_{[i, j]}, i, j \in \mathbb{Z}, i<j$, the sets of the compatible sequences:

$$
\begin{aligned}
\overline{\mathscr{E}} & =\left\{\varepsilon \in\{0, \ldots, u\}^{\mathbf{Z}}: B_{\varepsilon_{k} \varepsilon_{k+1}}=1, \forall k \in \mathbb{Z}\right\}, \\
\overline{\mathscr{C}}_{[i, j]} & =\left\{\varepsilon \in\{0, \ldots, u\}^{[i, j]}: B_{\varepsilon_{k} \varepsilon_{k+1}}=1, \forall k \in[i, j]\right\},
\end{aligned}
$$


where $[i, j]=\{k \in \mathbb{Z}: i \leq k \leq j\}$. We define $\mathscr{P}^{(n)}$ by

$$
\mathscr{P}^{(n)}=\varphi^{-n}\left(\mathscr{P}^{(0)}\right) \vee \cdots \vee \varphi^{n}\left(\mathscr{P}^{(0)}\right) .
$$

$\mathscr{P}^{(n)}$ is also a Markov partition, whose elements are parallelograms. $Q_{\varepsilon}^{(n)}=$ $\bigcap_{k=-n}^{n} \varphi^{-k}\left(Q_{\varepsilon_{k}}\right)$ for every $\varepsilon \in \overline{\mathscr{E}}_{[-n, n]}$. We have

$$
\operatorname{diam} \mathscr{P}^{(n)} \leq c^{\prime} \delta_{0} \lambda^{-n},
$$

where $c^{\prime}$ is a positive constant depending only on $\varphi$ (for the proof of (1.3), (1.4), and (1.6) see the proof of the lemma of [12, p. 106] and of lemmas 6.3 and 6.4 of $[1$, p. 28 and ff.]).

For every $\varepsilon \in \overline{\mathscr{E}}$ the set $\bigcap_{k=-\infty}^{+\infty} \varphi^{-k}\left(\bar{Q}_{\varepsilon_{k}}\right)$ contains a single point which we denote by $\pi(\varepsilon)$. The map $\pi$ is an isomorphism in the measure theoretical sense between the dynamical systems $\left(T^{2}, \varphi, m\right)$ and $(\overline{\mathscr{E}}, T, \mu)$, where $T$ is the shift on the sequences: $(T \varepsilon)_{i}=\varepsilon_{i+1}$ and $\mu$ is the stationary measure on an irreducible aperiodic Markov chain with state space $\{0, \ldots, u\}$ and transition probability matrix $P_{i j}=$ $m\left(\varphi\left(Q_{i}\right) \cap Q_{j}\right) / m\left(Q_{i}\right)$ (see the theorem of [12, p. 104]).

Now we will give some properties of the Markov partition $\mathscr{P}$ :

(P1) Since $\lambda>1$ and $\underline{0}$ is a fixed point for $\varphi$, it is easy to see that $\varphi\left(Q_{i}\right) \cap Q_{i} \neq \varnothing$ for $i=0, \ldots, 3$ and, consequently, $B_{i i}=1$ for $0 \leq i \leq 3$.

(P2) Let $p \in H(\underline{0})$. Then we can find a $k>0$ and a compatible sequence $\varepsilon \in \overline{\mathscr{E}}_{[-k, k]}$ such that $\varphi^{j}(\underline{p}) \in Q_{\varepsilon_{j}}$ for $j \in[-k, k]$ and $\varepsilon_{-k}, \varepsilon_{k} \in\{0, \ldots, 3\}$. This is a straightforward consequence of the definition of homoclinic points. Indeed, since $\lim _{|j| \rightarrow \infty} \varphi^{j}(p)=\underline{0}$, we can find $k$ such that $\varphi^{-k}(p)$ and $\varphi^{k}(p)$ belong to the interior of $\bigcup_{i=0}^{\overline{3}} \bar{Q}_{i}$. Let us consider the Markov partition $\mathscr{P}^{(k)}$ and choose an element $Q_{\varepsilon}^{(k)}$ such that $p \in \bar{Q}_{\varepsilon}^{(k)} ;$ then the sequence $\varepsilon$ has the required properties.

\section{Preliminary results}

Now we state the relation between symbolic dynamics of toral automorphisms and properties $(0.2)$ by giving some simple geometric arguments.

Let $P:[0,1] \rightarrow T^{2}$ be the lifting $P(x)=x \underline{e}^{u} / e_{1}^{u}, V^{u}=P([0,1]) \subset W^{u}(0), \Delta=$ $\left\{p \in T^{2}: a \leq p_{1} \leq b\right\}, \partial \Delta$ its boundary and $\chi_{\Delta}$ the characteristic function of $\Delta$. Since $\lambda$ is an eigenvalue of $A$, with eigenvector $\underline{e}^{u}=\left(e_{1}^{u}, e_{2}^{u}\right)$ we have $\lambda^{j} x=\left(A^{j} x \underline{e}^{u} / e_{1}^{u}\right)_{1}$, so that $\left\{\lambda^{j} x\right\}=\left(\varphi^{j}(P(x))\right)$, and

$$
P_{i}^{N}(k)=l\left(\left\{x \in[0,1]: \sum_{j=0}^{N-1} \chi_{\Delta}\left(\varphi^{j}(P(x))\right)=k\right\}\right) .
$$

Definition (2.1). For every $n \geq 0$, let $\tilde{\Sigma}_{n}$ be the sequence of parallelograms:

$$
\tilde{\Sigma}_{n}=\bigcup_{\underline{p} \in V^{u}} W_{c_{0} \lambda^{-n / 2}}^{s}(\underline{p}) \text {, }
$$

then, if $c_{0}$ is small enough and $q \in \tilde{\Sigma}_{n}$, for any $n>0 w(\underline{q})=V^{u} \cap W_{c_{0} \lambda^{-n / 2}}^{s}(\underline{q})$ is a point in $V^{u}$. Using (2.1) we get:

$$
P_{l}^{N}(k)=m\left(\left\{\underline{p} \in \tilde{\Sigma}_{n}: \sum_{j=0}^{N-1} \chi_{\Delta}\left(\varphi^{j}(w(\underline{p}))\right)=k\right\}\right) / m\left(\tilde{\Sigma}_{n}\right) .
$$


Definition (2.2). Let

$$
\begin{aligned}
S_{n} & =\left\{\varepsilon \in \overline{\mathscr{E}}_{[-n, n]}: \bar{Q}_{\varepsilon}^{(n)} \subset \tilde{\Sigma}_{n}\right\} \\
\Sigma_{n} & =\bigcup_{\varepsilon \in S_{n}} \bar{Q}_{\varepsilon}^{(n)}, \\
D_{n} & =\left\{\varepsilon \in \overline{\mathscr{E}}_{[-n, n]}: \bar{Q}_{\varepsilon}^{(n)} \subset \Delta\right\} \\
\Delta_{n} & =\bigcup_{\varepsilon \in D_{n}} \bar{Q}_{\varepsilon}^{(n)}
\end{aligned}
$$

and let $m_{n}$ be the sequence of probability measures on $T^{2}: m_{n}(A)=$ $m\left(A \cap \Sigma_{n}\right) / m\left(\Sigma_{n}\right)$ for any $A \subset T^{2} m$-measurable. Let $\nu_{n}^{N}$ be the sequence of functions on $T^{2}: \nu_{n}^{N}(\underline{p})=\sum_{j=0}^{N-1} \chi_{\Delta_{n}}\left(\varphi^{j}(\underline{p})\right) . E_{n}^{N}, D_{n}^{N}$ and $P_{n}^{N}$ will denote, respectively, the expectation, the variance and the probability distribution of $\nu_{n}^{N}$ with respect to $m_{n}$.

Proposition (2.3). Let $\omega$ be a positive real number, $n=[\omega \log N]$ and $C(\omega, N)=$ $\sup _{0 \leq k \leq N}\left|P_{l}^{N}(k)-P_{n}^{N}(k)\right|$. Then, for any $r>0$, there is $\bar{\omega}$ such that for $\omega>\bar{\omega}$,

$$
\lim _{N \rightarrow \infty} C(\omega, N) N^{r}=0 .
$$

Proof. Using (2.3) and (2.4) we get

$$
\begin{aligned}
\mid P_{i}^{N}(k) & -P_{n}^{N}(k) \mid \\
\leq & m\left(\left\{\underline{p} \in \tilde{\Sigma}_{n}: \sum_{j=0}^{N-1} \chi_{\Delta}\left(\varphi^{j}(w(\underline{p}))\right) \neq \sum_{j=0}^{N-1} \chi_{\Delta_{n}}\left(\varphi^{j}(\underline{p})\right)\right\}\right) / m\left(\tilde{\Sigma}_{n}\right) \\
& +\left|m\left(\left\{\underline{p} \in \tilde{\Sigma}_{n}: \nu_{n}^{N}(\underline{p})=k\right\}\right) / m\left(\tilde{\Sigma}_{n}\right)-P_{i}^{N}(k)\right|
\end{aligned}
$$

and, by the $\varphi$-invariance of the measure $m$, it follows that

$$
\begin{aligned}
\mid P_{l}^{N}(k) & -P_{n}^{N}(k) \mid \\
\leq & \left(\sum_{j=0}^{N-1} m\left(\left\{\underline{p} \in \varphi^{j}\left(\tilde{\Sigma}_{n}\right): \chi_{\Delta}\left(\varphi^{j}\left(w\left(\varphi^{-j}(\underline{p})\right)\right)\right) \neq \chi_{\Delta}(\underline{p})\right\}\right) / m\left(\tilde{\Sigma}_{n}\right)\right. \\
& +\left(\sum_{j=0}^{N-1} m\left(\left\{\varphi^{j}\left(\tilde{\Sigma}_{n}\right) \cap\left(\Delta \backslash \Delta_{n}\right)\right\}\right)\right) / m\left(\tilde{\Sigma}_{n}\right) \\
& \left.+\mid m\left(B \cap \tilde{\Sigma}_{n}\right) / m\left(\tilde{\Sigma}_{n}\right)-m\left(B \cap \Sigma_{n}\right) / m\left(\Sigma_{n}\right)\right),
\end{aligned}
$$

where $B=\left\{\underline{p} \in T^{2}: \nu_{n}^{N}(\underline{p})=k\right\}$. Let us consider the $j$ th term of the first sum on the right-hand-side of (2.7). We observe that if $\underline{p} \in \varphi^{j}\left(\tilde{\Sigma}_{n}\right)$ and $\chi_{\Delta}(\underline{p}) \neq$ $\chi_{\Delta}\left(\varphi^{j}\left(w\left(\varphi^{-j}(\underline{p})\right)\right)\right)$ then the segment joining $\underline{p}$ to $\varphi^{j}\left(w\left(\varphi^{-j}(\underline{p})\right)\right)$ has to intersect $\partial \Delta$. This segment is parallel to the stable direction contained in $\varphi^{j}\left(\tilde{\Sigma}_{n}\right)$ and of length less than $c_{0} \lambda^{-n / 2} \lambda^{-j}$. Thus we have

$$
\left\{\underline{p} \in \varphi^{j}\left(\tilde{\Sigma}_{n}\right): \chi_{\Delta}(\underline{p}) \neq \chi_{\Delta}\left(\varphi^{j}\left(w\left(\varphi^{-j}(\underline{p})\right)\right)\right)\right\} \subset \bigcup_{q \in \varphi^{j}\left(\tilde{\Sigma}_{n}\right) \cap \Delta \Delta} W_{c_{0} \lambda}^{s}{ }^{-n / 2-j}(\underline{q}) .
$$

On the other hand $\varphi^{j}\left(\tilde{\Sigma}_{n}\right)$ is a strip whose sides are parallel to the stable and unstable directions and have lengths bounded by $c_{0} \lambda^{-n / 2} \lambda^{-j}$ and $c_{1} \lambda^{j}$ respectively. Therefore $\varphi^{j}\left(\tilde{\Sigma}_{n}\right) \cap \partial \Delta$ is a union of at most $c_{1} \lambda^{j}$ segments of length $c_{2} \lambda^{-n / 2} \lambda^{-j}$. We can now 
evaluate the measure of the set appearing on the right-hand side of (2.8) by:

$$
\begin{aligned}
m\left(\bigcup_{\underline{q} \in \varphi^{j}\left(\tilde{\Sigma}_{n}\right) \cap \partial \Delta} W_{c_{0} \lambda^{-n / 2-j}}^{s}(\underline{q})\right) & \leq c_{1} \lambda^{j}\left(c_{3} \lambda^{-n / 2-j} \lambda^{-n / 2-j}\right) / c_{0}^{\prime} \lambda^{-n / 2} \\
& =c_{4} \lambda^{-n / 2-j},
\end{aligned}
$$

where $c_{0}^{\prime}, c_{3}$ and $c_{4}$ are positive constants depending only on $\varphi$ and $c_{0}$. The measure of the set appearing in the $j$ th term of the second sum on the right-hand-side of (2.7) can be estimated in a similar manner. Since the elements of the partition $\mathscr{P}^{(n)}$ have diameter less than $c^{\prime} \delta_{0} \lambda^{-n}$, the set $\Delta \backslash \Delta_{n}$ is contained inside the union of two vertical strips of width $c^{\prime} \delta_{0} \lambda^{-n}$. We have

$$
\varphi^{j}\left(\tilde{\Sigma}_{n}\right) \cap\left(\Delta \backslash \Delta_{n}\right) \subset \bigcup_{q \in \varphi^{j}\left(\tilde{\Sigma}_{n}\right) \cap \partial \Delta} W_{c_{s} \lambda^{-n}}^{u}(\underline{q})
$$

and, using the same arguments as in the preceding estimates we get from (2.10)

$$
\begin{aligned}
m\left(\varphi^{j}\left(\tilde{\Sigma}_{n}\right) \cap\left(\Delta \backslash \Delta_{n}\right)\right) / m\left(\tilde{\Sigma}_{n}\right) & \leq c_{1} \lambda^{j}\left(c_{5} \lambda^{-n / 2-j} \lambda^{-n}\right) / c_{0}^{\prime} \lambda^{-n / 2} \\
& =c_{6} \lambda^{-n-j} .
\end{aligned}
$$

Finally we estimate the last term of (2.7):

$$
\begin{aligned}
\mid m(B & \left.\cap \tilde{\Sigma}_{n}\right) / m\left(\tilde{\Sigma}_{n}\right)-m\left(B \cap \Sigma_{n}\right) / m\left(\Sigma_{n}\right) \mid \\
& \leq\left(m\left(B \cap \tilde{\Sigma}_{n}\right)-m\left(B \cap \Sigma_{n}\right)\right) / m\left(\tilde{\Sigma}_{n}\right)+m\left(B \cap \Sigma_{n}\right)\left(1 / m\left(\Sigma_{n}\right)-1 / m\left(\tilde{\Sigma}_{n}\right)\right) \\
& \leq 2\left(m\left(\tilde{\Sigma}_{n}\right)-m\left(\Sigma_{n}\right)\right) / m\left(\tilde{\Sigma}_{n}\right) \\
& \leq 2\left(c^{\prime} \delta_{0} \lambda^{-n}\left(c_{7}+\delta_{0} \lambda^{-n / 2}\right) / c_{0}^{\prime} \lambda^{-n / 2}=c_{8} \lambda^{-n / 2}\right.
\end{aligned}
$$

where $c_{8}$ is a positive constant depending only on $\varphi, \delta_{0}$ and $c_{0}$. By putting together the estimates (2.9), (2.11) and (2.12) we get

$$
\left|P_{I}^{N}(k)-P_{n}^{N}(k)\right| \leq\left(c_{4} \lambda^{-n / 2}+c_{6} \lambda^{-n}\right) \lambda /(\lambda-1)+c_{8} \lambda^{-n / 2},
$$

so that for $\omega$ large enough we obtain (2.5).

From now on we shall assume $n=[\omega \log N]$, where $\omega$ is such that (2.5) is true for $r=4$.

Using proposition 2.3 we also obtain

$$
\left|E_{l}^{N}-E_{n}^{N}\right| \leq\left|\sum_{k=0}^{N-1} k\left(P_{l}^{N}(k)-P_{n}^{N}(k)\right)\right| \leq N^{2} C(\omega, N)
$$

and

$$
\begin{aligned}
\left|D_{l}^{N}-D_{n}^{N}\right| & \leq\left|\sum_{k=0}^{N-1} k^{2}\left(P_{l}^{N}(k)-P_{n}^{N}(k)\right)-\left(E_{l}^{N}\right)^{2}+\left(E_{n}^{N}\right)^{2}\right| \\
& \leq 3 N^{3} C(\omega, N) .
\end{aligned}
$$

For every $\varepsilon \in \overline{\mathscr{E}}$, let $\xi_{i}^{(n)}(\varepsilon)=\chi_{\Delta_{n}}\left(\varphi^{i}(\pi(\varepsilon))\right)$ and $\xi_{i}(\varepsilon)=\chi_{\Delta}\left(\varphi^{i}(\pi(\varepsilon))\right) . \xi_{i}^{(n)}$ is a cylinder function with base $[i-n, i+n]$. For any $\zeta \in \overline{\mathscr{E}}_{[-n, n]}$ we denote by $\mu_{\zeta}$ the measure $\mu$ conditioned to $\varepsilon_{i}=\zeta_{i}$ for $i \in[-n, n]$ and by $E_{\zeta}^{N}, D_{\zeta}^{N}, P_{\zeta}^{N}$, respectively, the : expectation, the variance and the probability distribution of $\sum_{i=0}^{N-1} \xi_{i}^{(n)}$ with respect 
to $\mu_{\zeta}$. For any $E \subset T^{2}$ we have:

$$
\begin{aligned}
m_{n}(E) & =m\left(E \cap \Sigma_{n}\right) / m\left(\Sigma_{n}\right) \\
& =\sum_{\zeta \in S_{n}} m_{n}\left(Q_{\zeta}^{(n)}\right)\left(m\left(E \cap Q_{\zeta}^{(n)}\right) / m\left(Q_{\zeta}^{(n)}\right)\right) \\
& =\sum_{\zeta \in S_{n}} m_{n}\left(Q_{\zeta}^{(n)}\right) \mu_{\zeta}\left(\pi^{-1}(E)\right),
\end{aligned}
$$

i.e. the measure $m_{n}$ of a set in $T^{2}$ can be evaluated as a convex combination of measures $\mu_{\zeta}$ of the corresponding set in $\overline{\mathscr{E}}$. The following proposition is an immediate consequence of $(2.5),(2.14),(2.15)$ and (2.16).

Proposition (2.4). Assume that uniformly with respect to $\zeta \in \overline{\mathscr{E}}_{[-n, n]}$ :

(i) $\lim _{N \rightarrow \infty} D_{\zeta}^{N} / N=\sigma^{2}>0$;

(ii) $\lim _{N \rightarrow \infty}\left|E_{n}^{N}-E_{\zeta}^{N}\right| N^{2}=\lim _{N \rightarrow \infty}\left|D_{n}^{N}-D_{\zeta}^{N}\right| N^{2}=0$;

(iii) $\lim _{N \rightarrow \infty} \sup _{0 \leq k \leq N}\left[\sqrt{D_{\zeta}^{N}} P_{\zeta}^{N}(k)-\exp \left(-\left(k-E_{\zeta}^{N}\right)^{2} / 2 D_{\zeta}^{N}\right)\right] / \sqrt{2 \pi}=0$.

Then (0.2) holds.

In order to prove $(0.2)$ therefore we need only to prove $(2.17)$. The proof will be demonstrated in $\S 3$, where we will make use of the estimates given in the following propositions.

PROPOSITION (2.5). Let $r, s \in \mathbb{Z}, r<s$ and let $\mathscr{F}_{[r, s]}, \mathscr{G}_{[r, s]}^{(n)}$ be the $\sigma$-algebras generated respectively by $\varepsilon_{i}, \xi_{i}^{(n)}, i \in[r, s]$. Then there exist two positive constants $\delta \in(0,1), \sigma_{1}$ such that uniformly in $N$ :

$$
\sup _{\substack{A \in \mathscr{F}_{[r-N, r]} \\ B \in \mathscr{F}_{(r+k, r+k+N]}}}|\mu(A \cap B)-\mu(A) \mu(B)| \leq \sigma_{1} \delta^{k},
$$

and two positive constants $\eta \in(0,1), \sigma_{2}$ such that, uniformly in $N$ and $n$ :

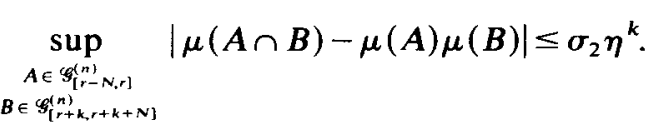

Proof. The proof of (2.18) can be obtained by standard procedure using the properties of the Markov chain (see e.g. [7, pp. 365, 366]). Property $(2.19)$ is a consequence of (2.18) and of the fact that:

$$
\begin{aligned}
\mu\left(\left\{\xi_{i}^{(n)} \neq \xi_{i}^{(k)}\right\}\right) & =m\left(\Delta_{n} \Delta \Delta_{k}\right) \leq m\left(\Delta \backslash \Delta_{\min (n, k)}\right) \\
& \leq 4 \delta_{0} \lambda^{-\min (n, k)} .
\end{aligned}
$$

The proof is completely similar to that of $[2,(1.26)$, p. 38].

Definition (2.6). Let $r, s \in \mathbb{Z}, r<s, \sigma, \sigma^{\prime} \in\{0, \ldots, u\}$. We denote by $B_{r, s}^{(n)}\left(\sigma, \sigma^{\prime}\right)$ the cylinder set, with base $[r-2 n, r] \cup[s, s+2 n]$ defined by:

$$
\begin{aligned}
B_{r, s}^{(n)}\left(\sigma, \sigma^{\prime}\right)= & \left\{\varepsilon \in \overline{\mathscr{E}}: \varepsilon_{r}=\sigma, \varepsilon_{s}=\sigma^{\prime} \text { and } \forall \eta \in \overline{\mathscr{E}} \text { with } \eta_{j}=\varepsilon_{j},\right. \\
& \left.j \notin[r+1, s-1], \xi_{i}^{(n)}(\eta)=\xi_{i}^{(n)}(\varepsilon), \text { for } i \in[r-n, r] \cup[s, s+n]\right\} .
\end{aligned}
$$

If $\varepsilon \in B_{r, s}^{(n)}\left(\sigma, \sigma^{\prime}\right)$, we need only to look at the symbols $\varepsilon_{i}$ for $i \notin[r-1, s-1]$ in order to know the values of $\xi_{i}^{(n)}$ for $i \in[r-n, r] \cup[s, s+n]$. 
Proposition (2.7). If $\delta_{0}$ is small enough, there are $\alpha>0, \bar{k} \in \mathbb{Z}^{+}$and at least two indices $\sigma_{1}, \sigma_{2} \in\{0, \ldots, 3\}$, such that for every $n$

$$
\mu\left(B_{r, s}^{(n)}\left(\sigma, \sigma^{\prime}\right)\right) \geq \alpha \quad \text { if } s-r>\bar{k}, \sigma, \sigma^{\prime} \in\left\{\sigma_{1}, \sigma_{2}\right\} .
$$

Proof. Let $B_{+}^{(n)}(\sigma), B_{-}^{(n)}(\sigma)$ be the events:

$$
\begin{aligned}
B_{+(-)}^{(n)}(\sigma)= & \left\{\varepsilon \in \overline{\mathscr{E}}: \varepsilon_{0}=\sigma \text { and } \forall \eta \in \overline{\mathscr{E}} \text { with } \eta_{j}=\varepsilon_{j}, j \geq 0,(j \leq 0),\right. \\
& \left.\xi_{i}^{(n)}(\eta)=\xi_{i}^{(n)}(\varepsilon), \forall i \in[0, n](i \in[-n, 0])\right\}
\end{aligned}
$$

It is easy to see that $B_{+}^{(n)}(\sigma) \in \mathscr{F}_{[0,2 n]}, B_{-}^{(n)}(\sigma) \in \mathscr{F}_{[-2 n, 0]}$ and $B_{r, s}^{(n)}\left(\sigma, \sigma^{\prime}\right) \supset T^{r} B_{-}^{(n)}(\sigma) \cap$ $T^{s} B_{+}^{(n)}\left(\sigma^{\prime}\right)$. Using the stationarity of $\mu$ and (2.18) of proposition (2.5) we get:

$$
\begin{aligned}
\mu\left(B_{r, s}^{(n)}\left(\sigma, \sigma^{\prime}\right)\right) & \geq \mu\left(T^{r} B_{-}^{(n)}(\sigma) \cap T^{s} B_{+}^{(n)}\left(\sigma^{\prime}\right)\right) \\
& \geq \mu\left(B_{-}^{(n)}(\sigma)\right) \mu\left(B_{+}^{(n)}\left(\sigma^{\prime}\right)\right)-\sigma_{1} \delta^{s-r} .
\end{aligned}
$$

Therefore we need only estimate from below $\mu\left(B_{-}^{(n)}(\sigma)\right)$ and $\mu\left(B_{+}^{(n)}\left(\sigma^{\prime}\right)\right)$ in order to prove (2.21). Since the two estimates are completely similar, we just deal with the case of $B_{+}^{(n)}\left(\sigma^{\prime}\right)$. We choose $\sigma \in\{0, \ldots, 3\}$ such that $0 \in \bar{Q}_{\sigma}, Q_{\sigma} \cap \partial \Delta=\varnothing$. (It is easy to see that if $\delta_{0}$ is small enough, there are at least two such elements.) Given $k \in \mathbb{Z}^{+}$we can find $\delta_{0}$ so small that, if $\operatorname{diam} \mathscr{P}^{(0)}<\delta_{0}, \chi_{\Delta_{n}}$ is constant on $\bigcup_{0 \leq j \leq k} \varphi^{j}\left(Q_{\sigma}\right)$ for every $n \geq 0$ (see the properties of $\mathscr{P}^{(0)}$ given in $\S 1$ ). This means that $\xi_{i}^{(n)}(\eta)=\xi_{i}^{(n)}(\varepsilon)$ for $0 \leq i \leq k$ if $\eta_{0}=\varepsilon_{0}=\sigma$. We define

$$
H_{j}^{(n)}(\sigma)=\left\{\varepsilon \in \overline{\mathscr{E}}: \varepsilon_{0}=\sigma \text { and } \exists \eta \in \overline{\mathscr{E}} \text {, with } \eta_{i}=\varepsilon_{i}, i \geq 0 \text { such that } \xi_{j}^{(n)}(\eta) \neq \xi_{j}^{(n)}(\varepsilon)\right\} \text {. }
$$

In view of the previous remark, $H_{j}^{(n)}(\sigma)=\varnothing$ for $0 \leq j \leq k$, we have

$$
\pi^{-1}\left(Q_{\sigma}\right) / B_{+}^{(n)}(\sigma)=\bigcup_{j=0}^{\infty} H_{j}^{(n)}(\sigma)=\bigcup_{j=k+1}^{n} H_{j}^{(n)}(\sigma) .
$$

Now we evaluate $\mu\left(H_{j}^{(n)}(\sigma)\right)$ for $k<j \leq n$. If $\varepsilon, \eta \in \overline{\mathscr{E}}$ and $\varepsilon_{i}=\eta_{i}$ for $i \geq 0$, then $\pi(\eta) \in W_{\delta_{0}}^{s}(\pi(\varepsilon))$, so that $\xi_{j}^{(n)}(\eta) \neq \xi_{j}^{(n)}(\varepsilon)$ implies $\varphi^{j}\left(W_{\delta_{0}}^{s}(\pi(\varepsilon))\right) \cap \partial \Delta_{n} \neq \varnothing$. Therefore we have

$$
\pi\left(H_{j}^{(n)}(\sigma)\right) \subset \bigcup_{\underline{p} \in \varphi^{j}\left(\bar{Q}_{\sigma}\right) \cap \partial \Delta_{n}} W_{\delta_{0} \lambda^{-j}}^{s}(\underline{p}) \subset \bigcup_{\underline{p} \in \varphi^{j}\left(\bar{Q}_{\sigma}\right) \cap \Delta_{n}^{j}} W_{\delta_{0} \lambda^{-j}}^{s}(\underline{p}),
$$

where $\Delta_{n}^{\prime}=\left\{q \in \Delta: d(q, \partial \Delta) \leq \delta_{0} \lambda^{-n}\right\}$.

By using the same arguments as in proposition (2.3), one can see that $\varphi^{j}\left(\bar{Q}_{\sigma}\right) \cap \Delta_{n}^{\prime}$ is contained in the union of at most $c_{1} \lambda^{j}$ parallelograms with sides of length $c_{9} \delta_{0} \lambda^{-n}$ and $c_{10} \delta_{0} \lambda^{-j}\left(c_{9}, c_{10}\right.$ are constants depending only on $\left.\varphi\right)$. Therefore:

$$
\begin{aligned}
\mu\left(H_{j}^{(n)}(\sigma)\right) & \leq m\left(\bigcup_{\underline{p} \in \varphi^{j}\left(\bar{Q}_{\sigma}\right) \cap \Delta_{n}^{\prime}} W_{\delta_{0} \lambda^{-j}}^{s}(\underline{p})\right) \\
& \leq c_{11} \delta_{0}^{2}\left(\lambda^{-j}+\lambda^{-n}\right) \leq 2 c_{11} \delta_{0}^{2} \lambda^{-j} .
\end{aligned}
$$

Hence by (2.23), (2.26) and (1.4) we get

$$
\begin{aligned}
\mu\left(B_{+}^{(n)}(\sigma)\right) & \geq m\left(\bar{Q}_{\sigma}\right)-\sum_{j=k}^{n} \mu\left(H_{j}^{(n)}(\sigma)\right) \\
& \geq \delta_{0}^{2}\left(c-2 c_{11} \lambda^{-k} /\left(1-\lambda^{-1}\right)\right),
\end{aligned}
$$


so that, for $k$ large enough,

$$
\mu\left(B_{+}^{(n)}(\sigma)\right) \geq c \delta_{0}^{2} / 2
$$

Proposition (2.8). Let $r, s \in \mathbb{Z}, r<s$. If $\delta_{0}$ is small enough, $\bar{k}=s-r$ is large enough and $\sigma \in\left\{\sigma_{1}, \sigma_{2}\right\}$ (see proposition (2.7)), then there is $d>0$ such that for any $n$

$$
\inf _{\substack{\zeta^{\prime} \in \overline{\mathcal{E}}_{\mathcal{G}_{r, n, r}} \\ \zeta^{\prime \prime} \in \overline{\mathcal{E}}_{[, s, s+n]} \\ \zeta_{r}^{\prime}=\zeta_{s}^{\prime}=\sigma}} D_{\mu}\left(\sum_{i=r}^{s} \xi_{i}^{(n)} \mid \varepsilon_{k}=\zeta_{k}^{\prime}, k \in[r-n, r], \varepsilon_{k}=\zeta_{k}^{\prime \prime}, k \in[s, s+n]\right) \geq d>0,
$$

where $D_{\mu}$ denotes the variance with respect to $\mu$.

Proof. If $\delta_{0}$ is small enough, one can choose $\sigma \in\left\{\sigma_{1}, \sigma_{2}\right\}, \sigma^{\prime} \in\{0, \ldots, u\}$ such that $\underline{0} \in \bar{Q}_{\sigma}$ and either $Q_{\sigma} \subset \Delta, Q_{\sigma^{\prime}} \subset T^{2} \backslash \Delta$ or $Q_{\sigma} \subset T^{2} \backslash \Delta, Q_{\sigma^{\prime}} \subset \Delta$. Moreover, if $s-r$ is large enough, we can find $i, j \geq 0$ such that $\varphi^{i}\left(Q_{\sigma}\right) \cap Q_{\sigma^{\prime}} \neq \varnothing, \varphi^{j}\left(Q_{\sigma^{\prime}}\right) \cap Q_{\sigma} \neq \varnothing$ and $i+j<s-r$. We define two sequences $\eta, \eta^{\prime} \in \overline{\mathscr{E}}_{[r, s]}$ in the following way: $\eta_{k}=\sigma$ for $k \in[r, s]$ (the sequence $\eta$ is compatible since $\varphi\left(Q_{\sigma}\right) \cap Q_{\sigma} \neq \varnothing$ ) and $\eta^{\prime}$ is any compatible sequence such that $\eta_{r}^{\prime}=\sigma, \eta_{r+i}^{\prime}=\sigma^{\prime}$ and $\eta_{k}^{\prime}=\sigma$ for $r+i+j \leq k \leq s$ (such a sequence exists because of the choice of $i$ and $j$ ). For every $\varepsilon, \varepsilon^{\prime} \in \overline{\mathscr{E}}$ which agree in the interval $[r, s]$ with $\eta$ and $\eta^{\prime}$ respectively, we have either

or

$$
\sum_{k=r}^{s}\left(\xi_{k}^{(n)}(\varepsilon)-\xi_{k}^{(n)}\left(\varepsilon^{\prime}\right)\right) \geq 1, \quad \text { if } Q_{\sigma} \subset \Delta,
$$

$$
\sum_{k=r}^{s}\left(\xi_{k}^{(n)}(\varepsilon)-\xi_{k}^{(n)}\left(\varepsilon^{\prime}\right)\right) \leq-1, \quad \text { if } Q_{\sigma} \subset T^{2} \backslash \Delta,
$$

since $\xi_{k}^{(n)}(\varepsilon)$ is constant for $k \in[r, s]$ and $\xi_{r+i}^{(n)}(\varepsilon) \neq \xi_{r+i}^{(n)}\left(\varepsilon^{\prime}\right)$. We obtain the proposition by estimating

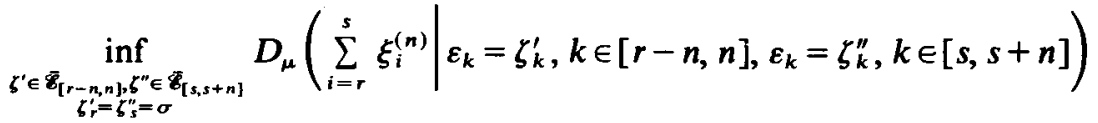

$$
\begin{aligned}
& \geq \frac{1}{4} \min \left(\mu\left(\left\{\varepsilon \in \overline{\mathscr{E}}: \varepsilon_{k}=\eta_{k}, k \in[r, s]\right\}\right), \mu\left(\left\{\varepsilon \in \overline{\mathscr{E}}: \varepsilon_{k}=\eta_{k}^{\prime}, k \in[r, s]\right\}\right)\right) .
\end{aligned}
$$

Equation (2.31) follows from (2.30) and an elementary estimate of the conditional variance.

Proposition (2.9). For a dense open set of intervals $[a, b] \subset[0,1]$ one can choose $\delta_{0}$ so small, $\sigma, \sigma^{\prime} \in\left[\sigma_{1}, \sigma_{2}\right]$ (see proposition (2.7)), $\bar{k}$ so large, that for $r, s \in \mathbb{Z}, s-r \geq \bar{k}$ there exists $\beta$, depending only on $s-r$, such that for $m \geq 2$ and $n \geq 0$ :

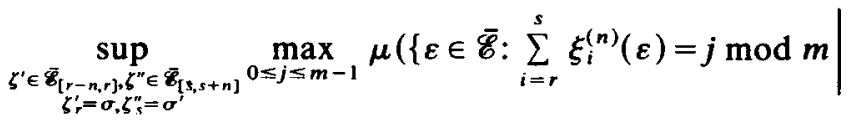

$$
\begin{aligned}
& \left.\left.\varepsilon_{k}=\zeta_{k}^{\prime}, k \in[r-n, r], \varepsilon_{k}=\zeta_{k}^{\prime \prime}, k \in[s, s+n]\right\}\right)<1-\beta \text {. }
\end{aligned}
$$

Proof. Let us first assume that $[a, b]$ satisfies the hypotheses

(a) $\left(\left\{\underline{p} \in T^{2}: p_{1}=a\right\} \cup\left\{\underline{p} \in T^{2}: p_{1}=b\right\}\right) \cap H(\underline{0})=\partial \Delta \cap H(\underline{0}) \neq \varnothing$;

(b) $a \neq 0, b \neq 1$. 
The hypothesis (2.33(b)) is made just for the sake of simplicity; when it is verified, the thesis of proposition (2.7) is true for an arbitrary choice of $\sigma_{1}, \sigma_{2} \in\{0, \ldots, 3\}$. Let $\underline{h} \in H(\underline{0}) \cap \partial \Delta$, then $\underline{h}$ uniquely defines $h^{u}, h^{s} \in \mathbb{R}$ such that $\underline{h}=h^{u} \underline{e}^{u}(\bmod 1)$ and $\underline{h}=h^{s} \underline{e}^{s}(\bmod 1)$. Let $\underline{\tilde{c}}, \underline{\tilde{b}}, \underline{\underline{b}}^{\prime} \in \mathbb{R}^{2}$ be defined as:

(i) $\underline{\tilde{c}}=\left\{\xi \underline{e}^{u}, \xi \in \mathbb{R}\right\} \cap\left\{\underline{q}+\xi \underline{e}^{s}, \xi \in \mathbb{R}\right\}, \quad$ where $\underline{q}=(0,1) \in \mathbb{R}^{2}$;

(ii) $\underline{\hat{b}}=\underline{h}+A^{N-1} \underline{\tilde{c}}-A^{-N} \underline{\tilde{c}}$;

(iii) $\underline{\hat{b}}^{\prime}=\underline{h}+A^{N} \underline{\tilde{c}}-A^{-N+1} \underline{\tilde{c}}$;

where $N$ will be suitably chosen in the following. Let $\underline{c}, \underline{b}, \underline{b}^{\prime}$ be the points of $T^{2}$ corresponding to $\underline{\tilde{c}}, \underline{\tilde{b}}, \underline{\tilde{b}}^{\prime}$. With the notation $h^{+(-)}=h^{s(u)} e_{1}^{s(u)}, \varphi^{k}(\underline{b})=\left(b_{1}^{(k)}, b_{2}^{(k)}\right)$, $\varphi^{k}\left(b^{\prime}\right)=\left(b_{1}^{\prime(k)}, b_{2}^{\prime(k)}\right)$ it follows from the previous definitions and (2.34) that, for any $k$,

$$
\begin{aligned}
b_{1}^{(k)} & =h^{+} \lambda^{-k}+\tilde{c}_{1}\left(\lambda^{-|k+N-1|}-\lambda^{-|k-N|}\right)(\bmod 1) \\
& =h^{-} \lambda^{k}+\tilde{c}_{1}\left(\lambda^{-|k+N-1|}-\lambda^{-|k-N|}\right)(\bmod 1) \\
b_{1}^{\prime(k)} & =h^{+} \lambda^{-k}+\tilde{c}_{1}\left(\lambda^{-|k+N|}-\lambda^{-|k-N+1|}\right)(\bmod 1) \\
& =h^{-} \lambda^{k}+\tilde{c}_{1}\left(\lambda^{-|k+N|}-\lambda^{-|k-N+1|}\right)(\bmod 1)
\end{aligned}
$$

Before going on with the proof, we make some comments on the behaviour of the orbits of $\underline{b}$ and $\underline{b}^{\prime}$. We can see from $(2.34)$ and $(2.35)$ that $\varphi^{k}(b)$ and $\varphi^{k}\left(b^{\prime}\right)$, for $k$ small in comparison with $N$, are obtained by adding small perturbations to $\varphi^{k}(\underline{h})$. If we look at the first coordinates, which determine whether a point belongs to $\Delta$, we see that the perturbations have the same direction for $k \neq 0$, whereas for $k=0$ they have opposite directions. For $k= \pm N+k_{0}$, with $\left|k_{0}\right|$ small with respect to $N$, $\varphi^{k+1}(\underline{b})$ and $\varphi^{k}\left(\underline{b}^{\prime}\right)$ are close to $\varphi^{k_{0}}(\underline{c})$. For the other values of $k, \varphi^{k}(\underline{b})$ and $\varphi^{k}\left(\underline{b}^{\prime}\right)$ are both close to $\underline{0}$.

Precisely, for $N$ big enough, one can find integers $N_{1}, N_{2}, N_{3}$, with $N_{3}>N>N_{2}>$ $N_{1}$ such that:

$\left(h_{1}\right) \inf _{k \in \mathbb{Z}}\left\{d\left(\varphi^{k}(\underline{b}), \partial \Delta\right)\right\} \geq \rho>0 ; \inf _{k \in \mathbb{Z}}\left\{d\left(\varphi^{k}\left(\underline{b}^{\prime}\right), \partial \Delta\right)\right\} \geq \rho \geq 0$.

This follows, for $N$ large enough, from the fact that there is only a finite set of $k$, for which $\varphi^{k}(\underline{h})$ or $\varphi^{k}(\underline{c})$ are close to $\partial \Delta$. Moreover

$\left(\mathrm{h}_{2}\right) \chi_{\Delta}(\underline{b}) \neq \chi_{\Delta}\left(\underline{b}^{\prime}\right)$;

$\left(\mathrm{h}_{3}\right) \chi_{\Delta}\left(\varphi^{k}(\underline{b})\right)=\chi_{\Delta}\left(\varphi^{k}\left(\underline{b}^{\prime}\right)\right), \quad$ for $|k| \leq N_{1}, k \neq 0$.

In fact, $\chi_{\Delta}\left(\varphi^{k}(\underline{b})\right)=\chi_{\Delta}\left(\varphi^{k}\left(\underline{b}^{\prime}\right)\right)=\chi_{\Delta}\left(\varphi^{k}(\underline{h})\right)$ for those $k$ such that $\varphi^{k}(\underline{h}) \notin \partial \Delta$ and, if $k$ is such that $\varphi^{k}(\underline{h}) \in \partial \Delta,\left(h_{3}\right)$ is also true because the perturbations $\varphi^{k}(\underline{b}-\underline{h})$ and $\varphi^{k}\left(\underline{b}^{\prime}-\underline{h}\right)$ have the same direction. Next

$\left(h_{4}\right) \chi_{\Delta}\left(\varphi^{k}(\underline{b})\right)=\chi_{\Delta}\left(\varphi^{k}\left(\underline{b}^{\prime}\right)\right)=0$, for $N_{1} \leq|k| \leq N_{2}$.

For $N$ large enough, we can choose $N_{1}, N_{2}$ such that, for $N_{1} \leq|k| \leq N_{2}$, all the three terms in (2.35) are small (for example $N_{1}, N_{2} \simeq N / 2$ ). Furthermore

$\left(h_{5}\right) \chi_{\Delta}\left(\varphi^{k+1}(\underline{b})\right)=\chi_{\Delta}\left(\varphi^{k}\left(b^{\prime}\right)\right)$, for $N_{2} \leq|k| \leq N_{3}$.

This follows from an argument similar to the one used for $\left(h_{3}\right)$, but here it is the orbit of $c$ that is perturbed. Finally

$\left(\mathrm{h}_{6}\right) \chi_{\Delta}\left(\varphi^{k}(\underline{b})\right)=\chi_{\Delta}\left(\varphi^{k}\left(\underline{b}^{\prime}\right)\right)=0$, for $|k| \geq N_{3}$.

For every Markov partition $\mathscr{P}^{(0)}$ with diameter smaller than $\rho / 2$, we can find $\sigma$, $\sigma^{\prime} \in\{0, \ldots, 3\}$, such that $\bar{Q}_{\sigma} \cap \Delta=\bar{Q}_{\sigma^{\prime}} \cap \Delta=\varnothing$, and two sequences $\eta, \eta^{\prime} \in \overline{\mathscr{E}}_{\left[-N_{3}, N_{3}\right]}$ with $\eta_{-N_{3}}=\eta_{-N_{3}}^{\prime}=\sigma, \quad \eta_{N_{3}}=\eta_{N_{3}}^{\prime}=\sigma^{\prime}$ such that $\underline{b} \in \bigcap_{k=-N_{3}}^{N_{3}} \varphi^{-k}\left(Q_{\eta_{k}}\right)$ and $\underline{b}^{\prime} \in$ 
$\bigcap_{k=-N_{3}}^{N_{3}} \varphi^{-k}\left(Q_{\eta_{k}}\right)$. This is possible through use of (P2) of $\S 1$. So, given $r, s \in \mathbb{Z}$, $s-r \geq 2 N_{3}$, we can define two sequences $\tau, \tau^{\prime} \in \overline{\mathscr{E}}_{[r, s]} \tau_{r+i}=\eta_{-N_{3}+i}, \tau_{r+i}^{\prime}=\eta_{-N_{3}+i}^{\prime}$ for $0 \leq i \leq 2 N_{3}$ and $\tau_{r+i}=\tau_{r+i}^{\prime}=\sigma^{\prime}$ for $2 N_{3} \leq i \leq s-r$. It follows from $\left(\mathrm{h}_{1}\right)-\left(\mathrm{h}_{6}\right)$ and from the construction of $\tau$ and $\tau^{\prime}$ that if $\varepsilon, \varepsilon^{\prime} \in \overline{\mathscr{E}}$ and $\varepsilon_{i}=\tau_{i}, \varepsilon_{i}^{\prime}=\tau_{i}^{\prime}$ for $i \in[r, s]$, then for any $n \geq 0$

$$
\left|\sum_{i=r}^{s} \xi_{i}^{(n)}(\varepsilon)-\xi_{i}^{(n)}\left(\varepsilon^{\prime}\right)\right|=1 .
$$

Thus, for every fixed $\zeta^{\prime} \in \overline{\mathscr{E}}_{[r-n, r]}, \zeta^{\prime \prime} \in \overline{\mathscr{E}}_{[s, s+n]}$ with $\zeta_{r}^{\prime}=\sigma$ and $\zeta_{s}^{\prime \prime}=\sigma^{\prime}$, and for any integers $j, m, m \geq 2,0 \leq j \leq m-1$, we have

$$
\begin{aligned}
& \mu\left(\left\{\varepsilon \in \overline{\mathscr{E}}: \sum_{i=r}^{s} \xi_{i}^{(n)}(\varepsilon)=j \bmod m \mid \varepsilon_{i}=\zeta_{i}^{\prime}, i \in[r-n, n], \varepsilon_{i}=\zeta_{i}^{\prime \prime}, i \in[s, s+n]\right\}\right) \\
& \quad \leq 1-\min \left(\mu\left(\left\{\varepsilon \in \overline{\mathscr{E}}: \varepsilon_{i}=\tau_{i}, i \in[r, s]\right\}\right), \mu\left(\left\{\varepsilon \in \overline{\mathscr{E}}: \varepsilon_{i}=\tau_{i}^{\prime}, i \in[r, s]\right\}\right)\right) \\
& \quad \leq 1-\left(\inf _{i, j \in\{0, \ldots, u\}: B_{i j}=1} P_{i j}\right)^{s-r}
\end{aligned}
$$

Hence (2.32) is proved.

Since $H(\underline{0})$ is a dense set in $T^{2}$, given an interval $[a, b] \subset[0,1]$ there is another interval $\left[a^{\prime}, b^{\prime}\right]$ with $\max \left(\left|a-a^{\prime}\right|,\left|b-b^{\prime}\right|\right)$ arbitrarily small, such that for $\left[a^{\prime}, b^{\prime}\right]$ the hypotheses (2.33) hold. On the other side, if $[a, b]$ satisfies $(2.33)$ and $\left[a^{\prime \prime}, b^{\prime \prime}\right]$ is such that $\max \left(\left|a-a^{\prime \prime}\right|,\left|b-b^{\prime \prime}\right|\right)<\rho / 2$, where $\rho$ is the constant appearing in $\left(\mathrm{h}_{1}\right)$, then it follows immediately that the sequences $\tau, \tau^{\prime} \in \overline{\mathscr{E}}_{[r, s]}$ constructed for $[a, b]$ also work for $\left[a^{\prime \prime}, b^{\prime \prime}\right]$.

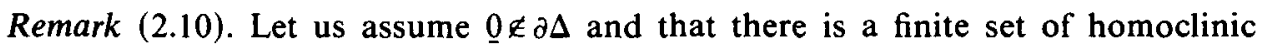
points $\underline{h}_{1}, \ldots, \underline{h}_{v}$ whose orbits keep away from $\partial \Delta$ and such that the largest common divisor of the $n_{i}=\sum_{k=-\infty}^{+\infty} \chi_{\Delta}\left(\varphi^{k} \underline{h}_{i}\right)$, for $i=1, \ldots, v$, is 1 . Then (2.32) is verified by considering compatible sequences corresponding to the points $\underline{h}_{1}, \ldots, \underline{h}_{v}$.

\section{Local limit theorem}

Assumptions (3.1). Throughout this section we will assume $\mathscr{P}^{(0)}, \vec{k}, \sigma_{1}, \sigma_{2} \in\{0, \ldots, 3\}$ are chosen in such a way that the conclusions of propositions (2.7), (2.8) and (2.9) are true with the constants $\alpha, d, \beta$, and $\omega$ as in 2.4. $E_{\mu}, D_{\mu}$ and $E_{\mu_{\zeta}}, D_{\mu_{\zeta}}$ will denote respectively the expectation and the variance with respect to $\mu$ and with respect to $\mu_{\zeta}, \zeta \in \overline{\mathscr{E}}_{[-n, n]}$.

Definitions (3.2). Let $p(N)$ and $q(N)$ be sequences of positive integers, $m=$ $[(N-n-1) /(p+q)]$ and

$$
\begin{array}{ccc}
J_{0}=[0, n], \quad J_{i}=[n+i(p+q), n+i(p+q)+q-1] & i=1, \ldots, m, \\
I_{i}=[n+(i-1)(p+q), n+(i-1)(p+q)+p-1] & i=1, \ldots, m, \\
\eta_{i}^{(n)}=\sum_{j \in I_{i}} \xi_{j}^{(n)}, \quad \zeta_{i}^{(n)}=\sum_{j \in J_{i}} \xi_{j}^{(n)} . &
\end{array}
$$

Lemma (3.3). Let $p(N)$ and $q(N)$ be such that $\lim \sqrt{N} / m=0$. Then for $\sigma, \sigma^{\prime} \in$ $\left\{\sigma_{1}, \sigma_{2}\right\}, p(N) \geq \bar{k}, 0<\alpha^{\prime}<\alpha$,

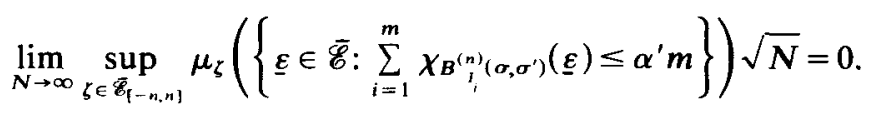


Proof. Let $\omega^{\prime}>0$. Using proposition (2.5) and (2.7) and applying Chebychev's inequality we get, for $N$ big enough,

$$
\begin{aligned}
& \mu_{\zeta}\left(\left\{\varepsilon \in \overline{\mathscr{E}}: \sum_{i=1}^{m} \chi_{B_{i}^{(n)}\left(\sigma, \sigma^{\prime}\right)}(\varepsilon) \leq \alpha^{\prime} m\right\}\right) \\
& \quad \leq \mu\left(\left\{\varepsilon \in \overline{\mathscr{E}}: \sum_{i: I_{i} \cap\left[0, n+\omega^{\prime} \log N\right]=\varnothing} \chi_{B_{i}^{(n)}\left(\sigma, \sigma^{\prime}\right)}(\varepsilon) \leq \alpha^{\prime} m\right\}\right)+\sigma_{1} \delta^{\omega^{\prime} \log N-2 n} \\
& \quad \leq c_{12} m /\left(\left(\alpha-\alpha^{\prime}\right) m-\left[\omega^{\prime} \log N\right]\right)^{2}+\sigma_{1} \delta^{\omega^{\prime} \log N-2 n},
\end{aligned}
$$

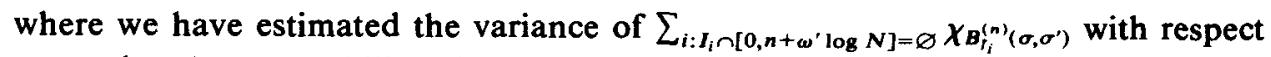
to $\mu$ using the summability of the mixing coefficients (2.18). The lemma is obtained by taking $\omega^{\prime}$ large enough.

LEMMA (3.4). There exists $\theta>0$ such that, if $N$ is large enough, $D_{\mu}\left(\sum_{i=0}^{N-1} \xi_{i}^{(n)}\right) \geq \theta N$.

Proof. Let $n_{0}=\left[\omega \log N_{0}\right]$ be given and $n \geq n_{0}$. Then

$$
\begin{aligned}
& \xi_{i}^{(n)}=\xi_{i}^{\left(n_{0}\right)}+\sum_{k=n_{0}}^{n-1}\left(\xi_{i}^{(k+1)}-\xi_{i}^{(k)}\right) \\
& D_{\mu}\left(\sum_{i=0}^{N-1} \xi_{i}^{(n)}\right) \\
& \geq D_{\mu}\left(\sum_{i=0}^{N-1} \xi_{i}^{\left(n_{0}\right)}\right) \\
& \quad-\sum_{k=n_{0}}^{n-1} \sum_{i, j=0}^{N-1}\left|E_{\mu}\left(\left(\xi_{i}^{\left(n_{0}\right)}-E_{\mu}\left(\xi_{i}^{\left(n_{0}\right)}\right)\right)\left(\xi_{i}^{(k+1)}-\xi_{i}^{(k)}-E_{\mu}\left(\xi_{i}^{(k+1)}-\xi_{j}^{(k)}\right)\right)\right)\right| \\
& \geq D_{\mu}\left(\sum_{i=0}^{N-1} \xi_{i}^{\left(n_{0}\right)}\right) \\
&-N \sum_{k=n_{0}}^{\infty} \sum_{j j \mid \leq n_{0}+2 k+2}\left|E_{\mu}\left(\left(\xi_{0}^{\left(n_{0}\right)}-E_{\mu}\left(\xi_{0}^{\left(n_{0}\right)}\right)\right)\left(\xi_{j}^{(k+1)}-\xi_{j}^{(k)}-E_{\mu}\left(\xi_{j}^{(k+1)}-\xi_{j}^{(k)}\right)\right)\right)\right| \\
&-N \sum_{k=n_{0}}^{\infty} \sum_{j j \mid>n_{0}+2 k+2}\left|E_{\mu}\left(\left(\xi_{0}^{\left(n_{0}\right)}-E_{\mu}\left(\xi_{0}^{\left(n_{0}\right)}\right)\right)\left(\xi_{j}^{(k+1)}-\xi_{j}^{(k)}-E_{\mu}\left(\xi_{j}^{(k+1)}-\xi_{j}^{(k)}\right)\right)\right)\right| \\
& \geq D_{\mu}\left(\sum_{i=0}^{N-1} \xi_{i}^{\left(n_{0}\right)}\right)-c_{13} N n_{0}\left(\max \left(\lambda^{-1}, \delta\right)\right)^{n_{0}} .
\end{aligned}
$$

The last inequality follows from $\left|\xi_{i}^{(k+1)}-\xi_{i}^{(k)}\right| \leq 1, \mu\left(\left\{\xi_{i}^{(k+1)} \neq \xi_{i}^{(k)}\right\}\right) \leq 4 \delta_{0} \lambda^{-k}$ (see (2.20) of proposition (2.5)) and (2.18) of proposition (2.5) (see e.g. [7, th. 17.2.1, p. 306]). Now let $q(N)=2 n_{0}+1, p(N)=\bar{k}$ and $\mathscr{T}_{N}=\mathscr{F}_{[-n, n]} \vee\left(\bigvee_{i=1}^{m} \mathscr{F}_{J_{i}}\right)$. It follows from propositions (2.7), (2.8) and lemma (3.3) that if $0<\alpha^{\prime}<\alpha, N$ is big enough and $A_{N}\left(\sigma, \sigma^{\prime}\right)$ is the event

then

$$
A_{N}\left(\sigma, \sigma^{\prime}\right)=\left\{\varepsilon \in \overline{\mathscr{E}}: \sum_{i=1}^{m} \chi_{B_{i}^{(n)}\left(\sigma, \sigma^{\prime}\right)}(\varepsilon)>\alpha^{\prime} m\right\},
$$

$$
\begin{aligned}
D_{\mu}\left(\sum_{i=0}^{N-1} \xi_{i}^{\left(n_{0}\right)}\right) & \geq E_{\mu}\left(\chi_{A_{N}}\left(\sigma, \sigma^{\prime}\right) D_{\mu}\left(\sum_{i=0}^{N-1} \xi_{i}^{\left(n_{0}\right)} \mid \mathscr{T}_{N}\right)\right) \\
& \geq \alpha^{\prime} m d \mu\left(A_{N}\left(\sigma, \sigma^{\prime}\right)\right) \geq c_{14}(N-n-1) /\left(2 n_{0}+\bar{k}+1\right) .
\end{aligned}
$$

The result follows from (3.4) and (3.5) for $n_{0}$ sufficiently large. 
We are now ready to prove the main result.

THEOREM (3.5). Let $[a, b]$ belong to the dense open set of subintervals that verify (2.32). Then (2.17) and hence (0.2) hold.

Proof. We know from proposition 2.4 that (2.17) implies (0.2). So we need only prove (2.17). Let us put:

$$
\sigma^{2}=D_{\mu}\left(\xi_{0}\right)+2 \sum_{j=1}^{\infty} E_{\mu}\left(\xi_{0} \xi_{j}-\left(E_{\mu}\left(\xi_{0}\right)\right)^{2}\right)=\lim _{N \rightarrow \infty} D_{\mu}\left(\sum_{j=0}^{N-1} \xi_{j}\right) / N
$$

Lemma 3.4 implies that $\sigma^{2}>0$.

From now on, we will use the notation: $E_{\mu}^{N}=E_{\mu}\left(\sum_{i=0}^{N-1} \xi_{i}^{(n)}\right), D_{\mu}^{N}=$ $D_{\mu}\left(\sum_{i=0}^{N-1} \xi_{i}^{(n)}\right)$. We give now some inequalities holding uniformly in $\zeta \in \overline{\mathscr{E}}_{[-n, n]}$ :

$$
\begin{gathered}
\left|E_{\zeta}^{N}-E_{\mu}^{N}\right| \leq c_{15} \log N \\
\left|D_{\zeta}^{N}-D_{\mu}^{N}\right| \leq c_{16}(\log N)^{2} \\
\left|D_{\mu}\left(\sum_{i \in I} \xi_{i}^{(n)}\right)-\sigma^{2}\right| I|| \leq c_{17}, \quad \text { for every interval } I \subset[0, N-1] \\
E_{\mu}\left(\left(\sum_{i \in I} \xi_{i}^{(n)}-|I| E_{\mu}\left(\xi_{0}^{(n)}\right)\right)^{4}\right) \leq c_{18}|I|^{2}, \quad \text { for every finite interval } I \subset \mathbb{Z} .
\end{gathered}
$$

(3.6) follows from (2.18) and the following inequality:

$$
\begin{aligned}
\left|E_{\zeta}^{N}-E_{\mu}^{N}\right| \leq & \left|\sum_{i=0}^{\left[\omega^{\prime} \log N\right]}\left(E_{\mu_{\xi}}\left(\xi_{i}^{(n)}\right)-E_{\mu}\left(\xi_{i}^{(n)}\right)\right)\right| \\
& +\left|E_{\mu_{\zeta}}\left(\sum_{i=\left[\omega^{\prime} \log N\right]^{\prime}+1}^{N-1} \xi_{i}^{(n)}\right)-E_{\mu}\left(\sum_{i=\left[\omega^{\prime} \log N\right]+1}^{N-1} \xi_{i}^{(n)}\right)\right| \\
\leq & 2 \omega^{\prime} \log N+N \sigma_{1} \delta^{\left(\omega^{\prime}-2 \omega\right) \log N},
\end{aligned}
$$

by choosing $\omega^{\prime}>2 \omega$. (3.8) is an easy consequence of proposition (2.5). (3.7) can be proved by using (3.8), proposition 2.5 and the following inequality:

$$
\begin{aligned}
\mid D_{\zeta}^{N}- & D_{\mu}\left(\sum_{i=0}^{N-1} \xi_{i}^{(n)}\right) \mid \\
\leq & 2\left|\sum_{\substack{i, j=0 \\
i<j}}^{N-1}\left(E_{\mu_{\zeta}}\left(\xi_{i}^{(n)} \xi_{j}^{(n)}\right)-E_{\mu_{\zeta}}\left(\xi_{i}^{(n)}\right) E_{\mu_{\zeta}}\left(\xi_{j}^{(n)}\right)-E_{\mu}\left(\xi_{i}^{(n)} \xi_{j}^{(n)}\right)+\left(E_{\mu}\left(\xi_{0}^{(n)}\right)\right)^{2}\right)\right| \\
\leq & 2 \sum_{i=0}^{2 n} \sum_{j=i}^{2(i+n)}\left(\left|E_{\mu_{\zeta}}\left(\xi_{i}^{(n)} \xi_{j}^{(n)}\right)-E_{\mu}\left(\xi_{i}^{(n)} \xi_{j}^{(n)}\right)\right|+\left|E_{\mu_{\zeta}}\left(\xi_{i}^{(n)}\right) E_{\mu_{\zeta}}\left(\xi_{j}^{(n)}\right)-\left(E_{\mu}\left(\xi_{0}^{(n)}\right)\right)^{2}\right|\right) \\
& +2 \sum_{i=2 n+1}^{\infty} \sum_{j=i}^{2(i+n)}\left(\left|E_{\mu_{\zeta}}\left(\xi_{i}^{(n)} \xi_{j}^{(n)}\right)-E_{\mu}\left(\xi_{i}^{(n)} \xi_{j}^{(n)}\right)\right|+\mid E_{\mu_{\zeta}}\left(\xi_{i}^{(n)}\right) E_{\mu_{\zeta}}\left(\xi_{j}^{(n)}\right)\right. \\
& \left.-\left(E_{\mu}\left(\xi_{0}^{(n)}\right)\right)^{2} \mid\right)+2 \sum_{i=0}^{\infty} \sum_{j=2(i+n)+1}^{\infty}\left(\left|E_{\mu_{\zeta}}\left(\xi_{i}^{(n)}\left(E_{\mu}\left(\xi_{j}^{(n)} \mid F_{[0, i+n]}\right)-E_{\mu_{\zeta}}\left(\xi_{j}^{(n)}\right)\right)\right)\right|\right. \\
& \left.\left.+\mid E_{\mu}\left(\xi_{i}^{(n)} \xi_{j}^{(n)}\right)-E_{\mu}\left(\xi_{0}^{(n)}\right)\right)^{2} \mid\right) \\
\leq & 8\left(4 n^{2}+n(2 n+1)\right)+4 \sum_{i=2 n+1}^{\infty} \sigma_{1}(1+2 n) \delta^{i}+2 \sum_{i=0}^{\infty} \sigma_{1} \sum_{k=i+1}^{\infty} \delta^{k} \\
& +2 \sum_{i=0}^{\infty} \sigma_{2} \sum_{k=i+2 n+1}^{\infty} \eta^{k} \\
\leq & c_{19}(\omega \log N)^{2} .
\end{aligned}
$$


(3.9) also follows from proposition (2.5) by developing the product (see e.g. the proof of lemma 18.5 .2 in [7, p. 348]).

(2.17 (i)) and (2.17 (ii)) follow from (3.6) and (3.7). In order to get (2.17 (iii)) let us make the following standard decomposition:

$$
\begin{aligned}
\sup _{0 \leq k \leq N} & \left(\sqrt{D_{\zeta}^{N}} P_{\zeta}^{N}(k)-\exp \left(-\left(k-E_{\zeta}^{N}\right)^{2} / 2 D_{\zeta}^{N}\right) / \sqrt{2 \pi}\right) \\
\leq & \int_{|t| \leq f_{1}(N)}\left|\varphi_{N, \zeta}(t)-\exp \left(-t^{2} / 2\right)\right| d t+\int_{|t|>f_{1}(N)} \exp \left(-t^{2} / 2\right) d t \\
& +\int_{f_{1}(N)<|t|<\gamma \sqrt{N}}\left|\varphi_{N, \zeta}(t)\right| d t+\int_{\gamma \sqrt{N}<|t|<\pi \sqrt{D_{\zeta}^{N}}}\left|\varphi_{N, \zeta}(t)\right| d t \\
= & I_{1}+I_{2}+I_{3}+I_{4},
\end{aligned}
$$

where

$$
\varphi_{N, \zeta}(t)=E_{\mu_{\zeta}}\left(\exp \left(i t\left(\sum_{k=0}^{N-1} \xi_{k}^{(n)}-E_{\mu_{\zeta}}\right) / \sqrt{D_{\zeta}^{N}}\right)\right) .
$$

We fix $f_{1}(N)=N^{1 / 8}$, whereas $\gamma$ will be suitably chosen in the sequel.

We start with the estimate of $I_{1}$. This estimate proves a generalized version of the integral limit theorem. We note that:

$$
\begin{aligned}
\mid \varphi_{N, \zeta}(t) & -E_{\mu}\left(\exp \left(i t\left(\sum_{k=0}^{N-1} \xi_{k}^{(n)}-E_{\mu}^{N}\right) / \sqrt{D_{\zeta}^{N}}\right)\right) \\
\leq & \mid E_{\mu_{\zeta}}\left(\exp \left(i t\left(\sum_{k=0}^{N-1} \xi_{k}^{(n)}-E_{\mu}^{N}\right) / \sqrt{D_{\zeta}^{N}}\right)\right) \\
& -E_{\mu}\left(\exp \left(i t\left(\sum_{k=0}^{N-1} \xi_{k}^{(n)}-E_{\mu}^{N}\right) / \sqrt{D_{\zeta}^{N}}\right)\right) \mid \\
& +\left|1-\exp \left(i t\left(E_{\zeta}^{N}-E_{\mu}^{N}\right) / \sqrt{D_{\zeta}^{N}}\right)\right| \\
\leq & 2 \omega^{\prime} \log N / \sqrt{\sigma^{2} N-c_{16}(\log N)^{2}}+\sigma_{1} \delta^{\left(\omega^{\prime}-2 \omega\right) \log N} \\
& +c_{15} \log N / \sqrt{\sigma^{2} N-c_{16}(\log N)^{2}},
\end{aligned}
$$

where we estimate the first term in the last inequality as in the proof of (3.6) and we have made use of (3.6) and (3.7). Then we can substitute $\varphi_{N, \zeta}$ with $E_{\mu}\left(\exp \left(i t\left(\sum_{k=0}^{N-1} \xi_{k}^{(n)}-E_{\mu}^{N}\right) / \sqrt{D_{\zeta}^{N}}\right)\right)$ in the integral $I_{1}$.

We now make the decomposition of definition (3.2) with the choice $p(N)=N^{7 / 10}$, $q(N)=N^{1 / 10}$ and put $\bar{\eta}_{i}^{(n)}=\eta_{i}^{(n)}-E_{\mu}\left(\eta_{i}^{(n)}\right), \bar{\zeta}_{i}^{(n)}=\zeta_{i}^{(n)}-E_{\mu}\left(\zeta_{i}^{(n)}\right)$. The sequences $p(N)$ and $q(N)$ are chosen in such a way that the $\bar{\zeta}_{i}^{(n)}$ 's can be neglected, whereas the $\bar{\eta}_{i}^{(n)}$ 's are so weakly dependent that we can apply to them a classical limit theorem for independent variables (see [7, Ch. 18]).

We have:

$$
\begin{aligned}
\left|E_{\mu}\left(\exp \left(i t\left(\sum_{k=0}^{N-1} \xi_{k}^{(n)}-E_{\mu}^{N}\right) / \sqrt{D_{\zeta}^{N}}\right)\right)-\left(E_{\mu}\left(\exp \left(i t \bar{\eta}_{1}^{(n)} / \sqrt{D_{\zeta}^{N}}\right)\right)\right)^{m}\right| \\
\leq E_{\mu}\left(\left|\exp \left(i t \sum_{k=0}^{N-1} \bar{\zeta}_{k}^{(n)} / \sqrt{D_{\zeta}^{N}}\right)-1\right|\right) \\
+\left|E_{\mu}\left(\exp \left(i t \sum_{k=0}^{N-1} \bar{\eta}_{k}^{(n)} / \sqrt{D_{\zeta}^{N}}\right)\right)-\left(E_{\mu}\left(\exp \left(i t \bar{\eta}_{1}^{(n)} / \sqrt{D_{\zeta}^{N}}\right)\right)\right)^{m}\right| .
\end{aligned}
$$


It follows from (2.9) of proposition (2.5) and from the inequalities (3.6), (3.7) and (3.8) (see $[7$, th. 18.4 .1 , p. 338]) that:

We can write:

$$
\begin{aligned}
\lim _{N \rightarrow \infty} N^{1 / 8} \sup _{\substack{|x| \leq N^{1 / 8} \\
\zeta \in \overline{\mathscr{E}}_{[-n, n]}}} \mid E_{\mu}\left(\exp \left(i t\left(\sum_{k=0}^{N-1} \xi_{k}^{(n)}-E_{\mu}^{N}\right) / \sqrt{D_{\zeta}^{N}}\right)\right) \\
-\left(E_{\mu}\left(\exp \left(i t \bar{\eta}_{1}^{(n)} / \sqrt{D_{\zeta}^{N}}\right)\right)\right)^{m} \mid=0
\end{aligned}
$$

$$
\begin{aligned}
& \left|\left(E_{\mu}\left(\exp \left(i t \bar{\eta}_{1}^{(n)} / \sqrt{D_{\zeta}^{N}}\right)\right)\right)^{m}-\exp \left(-t^{2} / 2\right)\right| \\
& \leq \mid\left(E_{\mu}\left(\exp \left(i \tau \bar{\eta}_{1}^{(n)} / \sqrt{m D_{\mu}\left(\bar{\eta}_{1}^{(n)}\right)}\right)\right)^{m}-\exp \left(-\tau^{2} / 2\right) \mid\right. \\
& \quad+\left|\exp \left(-\tau^{2} / 2\right)-\exp \left(-t^{2} / 2\right)\right|
\end{aligned}
$$

where $\tau=t\left(m D_{\mu}\left(\bar{\eta}_{1}^{(n)}\right) / D_{\zeta}^{N}\right)^{1 / 2}$.

The first term of the right-hand-side of (3.14) can be bounded by $\left(7 \tau^{3} / 6\right) \times$ $\left(E_{\mu}\left(\left|\bar{\eta}_{1}^{(n)}\right|^{3}\right) /\left(\sqrt{m}\left[D_{\mu}\left(\bar{\eta}_{1}^{(n)}\right)\right]^{3 / 2}\right) \cdot \exp \left(-\tau^{2} / 4\right)\right.$ using [5, theorem 2, p. 202]. So, by applying the estimates (3.7), (3.8) and (3.9) and inserting the expressions of $p, q$ and $m$ as functions of $N$ we get

$$
\sup _{\zeta \in \overline{\mathscr{E}}_{[-n, n]}} \lim _{N \rightarrow \infty} \int_{|t|<N^{1 / 8}}\left|\left(E_{\mu}\left(\exp \left(i t \bar{\eta}_{1}^{(n)} / \sqrt{D_{\zeta}^{N}}\right)\right)\right)^{m}-\exp \left(-t^{2} / 2\right)\right| d t=0
$$

The estimate of $I_{1}$ is obtained by combining (3.11), (3.13) and (3.15).

The estimate of $I_{2}$ is obvious. In order to estimate $I_{3}$ and $I_{4}$ we make the subdivisions of definition (3.2) with the choice $p(N)=\bar{k}, q(N)=2 n+1$ (see also the assumptions (3.1) for the constants that appear in the sequel). For $0<\alpha^{\prime}<\alpha$,

$$
\begin{gathered}
\mathscr{T}_{N}=\mathscr{F}_{[-n, n]} \vee\left(\bigvee_{i=0}^{m} \mathscr{F}_{J_{i}}\right), \quad \sigma, \sigma^{\prime} \in\left\{\sigma_{1}, \sigma_{2}\right\} \\
A_{N}\left(\sigma, \sigma^{\prime}\right)=\left\{\varepsilon \in \overline{\mathscr{E}}: \sum_{i=1}^{m} \chi_{B_{1}^{(n)}\left(\sigma, \sigma^{\prime}\right)}(\varepsilon)>\alpha^{\prime} m\right\}, \quad \zeta \in \overline{\mathscr{E}}_{[-n, n]},
\end{gathered}
$$

we get:

$$
\begin{aligned}
\left.\mid E_{\mu_{\zeta}}\left(\exp \left(i t\left(\sum_{k=0}^{N-1} \xi_{k}^{(n)}-E_{\zeta}^{N}\right) / \sqrt{D_{\zeta}^{N}}\right)\right)\right) \mid \\
=\left|E_{\mu_{\zeta}}\left(\exp \left(i t \sum_{k=0}^{N-1} \xi_{k}^{(n)} / \sqrt{D_{\zeta}^{N}}\right)\right)\right| \\
\leq\left|E_{\mu_{\zeta}}\left(E_{\mu}\left(\chi_{A_{N}\left(\sigma, \sigma^{\prime}\right)} \exp \left(i t \sum_{k=0}^{N-1} \xi_{k}^{(n)} / \sqrt{D_{\zeta}^{N}}\right) \mid \mathscr{T}_{N}\right)\right)\right| \\
+\left(1-\mu_{\zeta}\left(A_{N}\left(\sigma, \sigma^{\prime}\right)\right)\right)
\end{aligned}
$$

The fact that $\xi_{k}^{(n)}$ is a cylinder function, with base $[k-n, k+n]$, and the definition of $A_{N}\left(\sigma, \sigma^{\prime}\right)$ imply that for every $\varepsilon \in A_{N}\left(\sigma, \sigma^{\prime}\right)$ there are at least $\alpha^{\prime} m$ intervals $I_{i}$ such that $\eta_{i}^{(n)}$, only depends on $\varepsilon_{j}, j \in J_{i} \cup I_{i} \cup J_{i+1}$ and, for $k \notin I_{i}, \xi_{k}^{(n)}(\varepsilon)=\xi_{k}^{(n)}\left(\varepsilon^{\prime}\right)$, for all $\varepsilon^{\prime} \in \overline{\mathscr{E}}$ such that $\varepsilon_{j}^{\prime}=\varepsilon_{j}, j \notin I_{i}$. Then, by using the stationarity of $\mu$, we get:

$$
\begin{aligned}
& \left|E_{\mu_{\zeta}}\left(E_{\mu}\left(\chi_{A_{N}\left(\sigma, \sigma^{\prime}\right)} \exp \left(i t \sum_{k=0}^{N-1} \xi_{k}^{(n)} / \sqrt{D_{\zeta}^{N}}\right) \mid \mathscr{T}_{N}\right)\right)\right| \\
& \quad \leq \sup \left(\mid E_{\mu}\left(\exp \left(i t \eta_{1}^{(n)} / \sqrt{D_{\zeta}^{N}}\right)\left|\mathscr{T}_{N}, B_{I_{1}}^{(n)}\left(\sigma, \sigma^{\prime}\right)\right|\right)^{\alpha^{\prime} m} .\right.
\end{aligned}
$$


Now we make separate estimates for $t$ belonging to the regions corresponding to $I_{3}$ and $I_{4}$. By proposition (2.8) and Taylor's expansion of the characteristic function, there exists a constant $\gamma>0$ and $\sigma \in\left\{\sigma_{1}, \sigma_{2}\right\}$ such that

$$
\left|E_{\mu}\left(\exp \left(i t \eta_{1}^{(n)} / \sqrt{D_{\zeta}^{N}}\right) \mid \mathscr{T}_{N}, B_{I_{1}}^{(n)}\left(\sigma, \sigma^{\prime}\right)\right)\right| \leq \exp \left(-d t^{2} / 4 D_{\zeta}^{N}\right)
$$

for $|t| / \sqrt{D_{\zeta}^{N}}<\gamma$.

For the region corresponding to $I_{4}$ we can use (2.32) of proposition (2.9) to make an estimate of the characteristic function:

$$
\left|E_{\mu}\left(\exp \left(i t \eta_{1}^{(n)} / \sqrt{D_{\zeta}^{N}}\right) \mid \mathscr{T}_{N}, B_{l_{1}}^{(n)}\left(\sigma, \sigma^{\prime}\right)\right)\right| \leq e^{-c_{20},}
$$

for $\gamma \leq|t| / \sqrt{D_{\zeta}^{N}} \leq \pi$, where $c_{20}$ is a positive constant depending on $\beta$ and $\bar{k}$. We now prove the inequality (3.19). Let us fix a conditioning in $B_{I_{i}}^{(n)}\left(\sigma, \sigma^{\prime}\right)$ and denote by $p_{0}, \ldots, p_{\bar{k}}$ the conditional probabilities $\eta_{1}^{(n)}$ to assume the values $0,1, \ldots, \bar{k}$. We see from (2.32) that there are $k_{0}, \ldots, k_{d}$ with $0 \leq k_{0}<\cdots<k_{d} \leq \bar{k}, d \geq 1$ such that 1.c.d. $\left\{\left|k_{j}-k_{0}\right|, 1 \leq j \leq d\right\}=1$ and $\inf _{0 \leq j \leq d} p_{k_{j}} \geq \beta /(\bar{k}+1)$. Then there are $\gamma^{\prime}>0$, $1 \leq \bar{j} \leq d$, such that for every $\left.\tau \in[-\pi, \pi],|\tau|>\gamma, \mid \cos \left(\left(k_{\bar{j}}-k_{0}\right) \tau\right)-1\right) \mid>\gamma^{\prime}$ and

$$
\begin{aligned}
\left|\sum_{k=0}^{\bar{k}} p_{k} e^{i k \tau}\right| & =\left|\sum_{k=0}^{\bar{k}} p_{k} e^{i\left(k-k_{0}\right) \tau}\right| \\
& \leq \sum_{\substack{k=0 \\
k \neq k_{0}, k_{j}}}^{\bar{k}} p_{k}+\left(p_{k_{0}}+p_{k_{j}}\right)\left|p_{k_{0}} /\left(p_{k_{0}}+p_{k_{j}}\right)+p_{k_{j}} e^{i\left(k_{j}-k_{0}\right) \tau} /\left(p_{k_{0}}+p_{k_{j}}\right)\right| \\
& \leq 1-\beta \gamma^{\prime} /(\bar{k}+1) .
\end{aligned}
$$

Now, inserting in the right-hand-side of (3.16) the estimate (3.1) of lemma (3.3) and the inequalities (3.18), (3.19) and (3.20), we obtain that:

$$
\lim _{N \rightarrow \infty} I_{3}=0, \quad \lim _{N \rightarrow \infty} I_{4}=0 .
$$

The theorem is proved.

\section{Concluding remarks}

Integral theorems for sequences of random variables arising in number theory are proved, e.g., in [10] (limit theorem for the continued fraction expansion), in [6] for functions of the variables $\left\{\lambda^{n} x\right\}$ with $\lambda=2$. In the case $\lambda=2$, Moskvin and Postnikov [8] prove the local limit theorem. The same result is derived in a general context by Rousseau-Egele [11] by studying the spectral properties of the Ruelle-PerronFrobenius operator along the lines of the proof of the local limit theorem for Markov chains by Nagaev [9]. All these papers deal with the iterates of expanding maps. In our case, after the reduction to the symbolic dynamics on the torus, the variables are functions of all the sequence of symbols, whereas in the case of expanding maps of the interval they depend just on a half line and the arithmetical problem seems easier to treat.

Acknowledgements. We are grateful to Ya. G. Sinai, who suggested the problem to us. 


\section{REFERENCES}

[1] R. L. Adler \& B. Weiss. Similarity of automorphisms of the torus. Mem. Amer. Math. Soc. 98 (1970).

[2] R. Bowen. Equilibrium States and the Ergodic Theory of Anosov Diffeomorphisms. Lect. Notes in Math. 470. Springer-Verlag, 1975.

[3] I. P. Cornfeld, S. V. Fomin \& Ya. G. Sinai. Ergodic Theory. Springer Verlag, 1982.

[4] R. L. Dobrushin \& B. Tirozzi. The central limit theorem and the problem of equivalence of ensembles. Commun. Math. Phys. 54 (1977), 173-192.

[5] B. V. Gnedenko \& A. N. Kolmogorov. Limit Distributions for Sums of Independent Random Variables. Addison Wesley, 1954.

[6] I. A. Ibragimov. The central limit theorem for sums of functions of independent random variables and sums of the form $\sum_{k} f\left(2^{k} t\right)$. Theory Probab. Appl. 12 (1967), 596-607.

[7] 1. A. Ibragimov \& Yu. V. Linnik. Independent and Stationary Sequences of Random Variables. Noordhoff: Groningen, 1971.

[8] D. A. Moskvin \& A. G. Postnikov. A local limit theorem for the distribution of fractional parts of an exponential function. Theory Probab. Appl. 23 (1978), 521-528.

[9] S. V. Nagaev. Some limit theorems for homogeneous Markov chains. Theory Probab. Appl. 2 (1957) 378-406.

[10] W. Phillipp. Some metrical theorems in number theory, II. Duke Math. J. 37 (1970), 447-458.

[11] J. Rousseau-Egele. Un théorème de la limite locale pour une classe de transformations monotones par morceaux. Preprint.

[12] Ya. G. Sinai. Introduction to Ergodic Theory. Princeton Univ. Press, 1976. 